\title{
A Rare Cause of Late-Onset Epilepsy: Linear Scleroderma en Coup de Sabre
}

\author{
Haoyue Zhu, Xiuli Shang* \\ Department of Neurology, The First Affiliated Hospital of China Medical University, Shenyang, China \\ Email: urwelcomemoon@hotmail.com, "shang_zhao@sohu.com
}

Received 28 July 2015; accepted 23 April 2016; published 26 April 2016

Copyright $@ 2016$ by authors and Scientific Research Publishing Inc.

This work is licensed under the Creative Commons Attribution International License (CC BY).

http://creativecommons.org/licenses/by/4.0/

cc) (i)

\begin{abstract}
Late-Onset Epilepsy (LOE), with onset in adult life, is often attributed to cerebrovascular disease and intracranial tumor. Herein we present a LOE patient with history of Linear Scleroderma en Coup de Sabre (LScs) and abnormal cranial MRI signs. Curiously, his band-like skin lesion, presenting on the forehead, was in line with the surface projection of the intracranial focus shown in MRI. This gave a clue of the link between the skin lesion and the intracranial focus and the epilepsy. To sum up, it exposed a rare cause of LOE. Moreover, it underlined the significance of recognizing the cause to be associated with a substantially increased risk of developing epilepsy.
\end{abstract}

\section{Keywords}

Epilepsy, Late-Onset, Linear Scleroderma en Coup de Sabre

\section{Introduction}

Late-Onset Epilepsy (LOE), with onset in adult life, is often attributed to cerebrovascular disease and intracranial tumor [1]. While our patient indicated a rare cause, which is the Linear Scleroderma en Coup de Sabre (LScs). LScs, a special subtype of scleroderma, is a rare disorder characterized by a band-like region of dermal thickening and hardening restricted to the forehead, giving the resemblance of the skin lesion to the stroke of a sabre. Although it is generally regarded as an autoimmune disease, the definite etiology has never been convincingly established. LScs primarily affects the skin, and may also affect underlying tissue. So, neurologic symptoms are not infrequently to be seen [2], but the LOE is rare in LScs patients, especially with the ischemic foci shown in MRI.

${ }^{*}$ Corresponding author. 


\section{Case Report}

A 33-year-old man presented with a band-like skin lesion on his forehead (Figure 1(A)), was brought to our emergency department with complains of epilepsy. According to witness, the seizure was presented with loss of consciousness and flickering of the right mouth angle. There was no uprolling of eyes, incontinence or tongue bite then. The seizure stopped in around 20 seconds and the patient regained consciousness. Another seizure attack occurred in emergency department with features of loss of consciousness, uprolled eyes and twisted extremities. There was no incontinence or tongue bite. The patient regained consciousness in about 2 minutes after promptly getting intravenous diazepam. Neurological examination showed no other definite abnormalities except anomic aphasia and depressed left nasolabial groove. The patient underwent an electroencephalograph (EEG) examination and continuous spikes, and spike and waves were reported in left temporal region for about 2 mins 30 secs. A Magnetic Resonance Imaging (MRI) scan of the brain was performed and abnormalities (Figure 1(B)-(D)) were revealed, which were just subjacent to the skin lesion of the scalp. This gave a clue of the link between the skin lesion and the intracranial foci and the epilepsy. According to the patient's history, he was clinically diagnosed with Linear Scleroderma en Coup Sabre (LScs) at the age of 2, which was then a small reddish plaque on his left forehead. After admission, he underwent a biopsy specimen from the lesion area, and the findings (Figure 1(F)) were consistent with the histopathologic features of late fibrotic stage of LScs [3]. No previous similar case was reported in his family. Anticonvultary therapy was performed with levetiracetam 500 mg twice a day with current fairly good control of seizures.
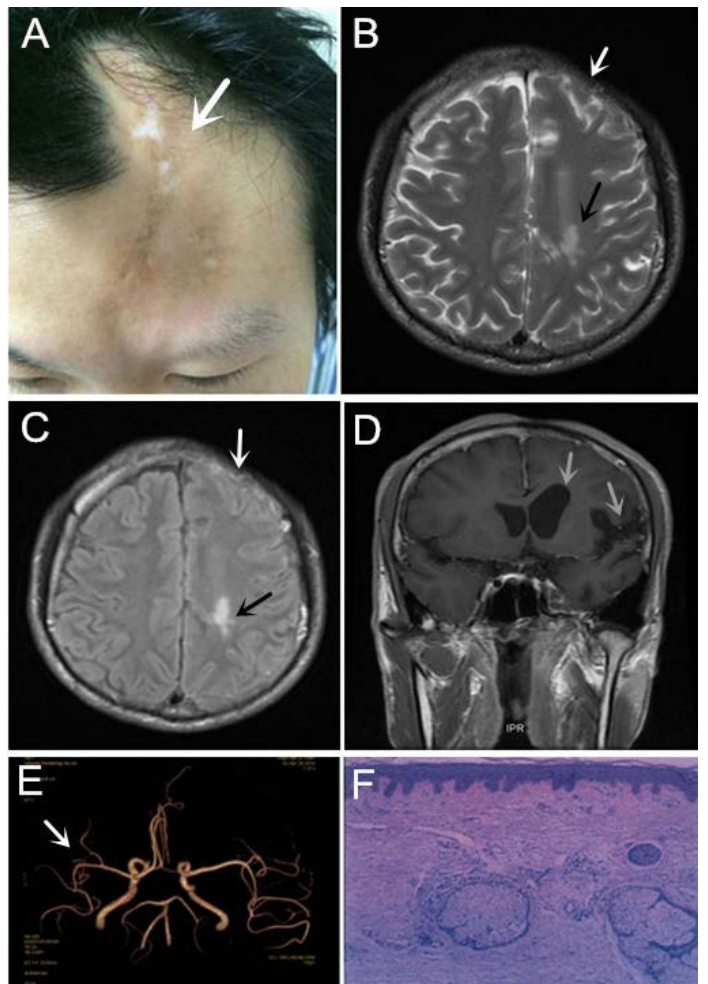

Figure 1. (A) A typical band-like, slightly depressed, $9 \mathrm{~cm} \times 4 \mathrm{~cm}$ indurated skin lesion (white arrow) overlying the left frontoparietal area was shown. It was hyperpigmented and associated with alopecia and hypesthesia, restricted to the forehead, and did not extend below the forehead; (B) Axial T2-weighted image showed left scalp atrophy (white arrow) with T2 hyperintensities in left semioval center (black arrow); (C) Axial fluidattenuated inversion recovery (FLAIR) image demonstrated left scalp atrophy (white arrow) with T2 hyperintensities in left semioval center (black arrow); (D) Coronal TI image demonstrated enlargement of left lateral ventricle and wider and deeper left lateral sulcus (gray arrow); (E) The left middle cerebral artery (MCA) shown in the magnetic resonance angiography (MRA) is slimmer (white arrow) compared to the right one, concurrent with the decreased distal vessels; (F) The biopsy specimen from the lesion area demonstrated that the upper $1 / 3$ of dermis was filled with tightly packed collagen fibers associated with cuticles and adnexal structures. Fat cells in the subcutaneous tissue were replaced by collagen. Small sweat glands were atrophic and surrounded by thickened collagen bundles. 


\section{Discussion}

Scleroderma encompasses a wide range of diseases from diffuse (Systemic Scleroderma, SS) to localized (Localized Scleroderma, LS) cutaneous fibrosis presentation, which is characterized by dermal thickening and hardening due to increased collagen deposition. Visceral fibrosis is visible in SS but not in LS. When the lesion of LS is restricted to the forehead given the resemblance of the skin lesions to the stroke of a sabre, it is referred as Linear Scleroderma en Coup de Sabre (LScs) [4]. LScs usually occurs in the first or second decades of life, and predominantly affects females. It slowly progresses for 2 to 5 years, preceded by stable period. Although it is generally regarded as an autoimmune disease, the definite etiology has never been convincingly established. LScs primarily affects the skin, and may also affect underlying tissue. So, neurologic symptoms are not infrequently to be seen, notably pediatric epilepsy [5], with subcortical calcification as the most common MRI sign [2].

To the best of our knowledge, the LOE is rare in LScs patients, especially with the ischemic foci shown in MRI. According to the previous reports, ischemic foci was not the main sign of cranial MRI, only that Kanzato $\mathrm{N}$ reported a localized scleroderma case in 1999, in which one the progressing ischemic stroke was associated and autoimmune pathogenesis of cerebrovascular injury was postulated as the possible reason [6]. For the sake of approaching the reason of the ischemic foci in our case, a Magnetic Resonance Angiography (MRA) was performed with scarcity of typical change of vasculities to stand by the immunological hypothesis although it seems to be the most generally accepted one by now. But we noticed that the left Middle Cerebral Artery (MCA) shown in the MRA is slimmer (Figure 1(E)) compared to the right hemisphere, concurrent with the decreased distal vessels. Taken together, the hypothesis which seems to be capable of coincidently interpreting both the cerebral atrophy and the slimmer vessels is a distinct one [7], which believes in that an early defect affecting one side of the rostral neural tube can cause facial and cerebral ipsilateral lesions subsequently, since these tissues have a common cell progenitor. Given the small sizes of the ischemic foci, they might arise from the thrombosis of perivasculars. Although no responsible stenosis of the vessels was found, the slimmer left MCA could have played a role more or less. Since the stroke has been shown to be the cause of $10 \%-15 \%$ of epilepsy [8], we speculated the ischemic foci to be remarkably associated with a substantially increased risk of developing epilepsy in our patient, and occurred in adulthood. In turn, this could also be one of the possible mechanisms underlying the epileptic presentation in LScs patients.

Due to the rarity of the cases, the evaluation of therapeutic benefit is limited. Currently much deficits exist in the treatment, and standard guideline is still absent. After the use of topical steroid and vitamin supplements, the lesion of our patient remained stable till maturity. Although steroid is one of the relatively accepted useful drugs for such lesions [3], it is still insensible to judge the stability solely owning to drug effect, or self-limited course of the lesion [2], or both. After admission, the patient was seizure free after levetiracetam treatment, but it is rather arbitrary to evaluate its effect now because of the refractoriness and intractability of the epilepsy caused by LSCS [2].

\section{Conclusion}

The patient was brought to our department due to seizures, and the initial reason was deduced to be associated with LScs. This case indicated that the cautiousness for the epileptic reason seeking is never excess, especially in adults. LScs complicated with epilepsy is not rare, but our case argued for the skepticism of the mechanism searching. Depending on the localized nature and nonprogressive course [9], LScs carries a relatively favorable prognosis, but affected central nervous system may limit the functional outcome.

\section{Acknowledgements}

We are very grateful to the patient for his cooperation and written consent for publication of his details and figures. We acknowledge with gratitude the efforts of Dr. Rajeev Ojha, MD. Neurology (Bir Hospital National Academy of Medical Science, Kathmandu, Nepal) in revising the manuscript.

\section{Informed Consent Form}

This study is not funded by any institution. Haoyue Zhu reports no disclosures. Xiuli Shang reports no disclosures. 


\section{Conflict of Interest}

None.

\section{Contributions of the Writers}

Haoyue Zhu contributed data collection and composition of this manuscript. Xiuli Shang contributed revision of this manuscript.

\section{References}

[1] Hanby, M.F., Al-Bachari, S., Makin, F., Vidyasagar, R., Parkes, L.M. and Emsley, H.C. (2015) Structural and Physiological MRI Correlates of Occult Cerebrovascular Disease in Late-Onset Epilepsy. NeuroImage Clinical, 9, 128-133. http://dx.doi.org/10.1016/j.nicl.2015.07.016

[2] Amaral, T.N., Peres, F.A., Lapa, A.T., Marques-Neto, J.F. and Appenzeller, S. (2013) Neurologic Involvement in Scleroderma: A Systematic Review. Seminars in Arthritis and Rheumatism, 43, 335-347. http://dx.doi.org/10.1016/j.semarthrit.2013.05.002

[3] Kreuter, A., Krieg, T., Worm, M., et al. (2009) [AWMF Guideline No. 013/066. Diagnosis and Therapy of Circumscribed Scleroderma]. Journal Der Deutschen Dermatologischen Gesellschaft, 7, S1-S14.

[4] Holland, K.E., Steffes, B., Nocton, J.J., Schwabe, M.J., Jacobson, R.D. and Drolet, B.A. (2006) Linear Scleroderma en Coup de Sabre with Associated Neurologic Abnormalities. Pediatrics, 117, e132-e136. http://dx.doi.org/10.1542/peds.2005-0470

[5] Zulian, F., Vallongo, C., Woo, P., et al. (2005) Localized Scleroderma in Childhood Is Not Just a Skin Disease. Arthritis \& Rheumatology, 52, 2873-2881. http://dx.doi.org/10.1002/art.21264

[6] Kanzato, N., Matsuzaki, T., Komine, Y., et al. (1999) Localized Scleroderma Associated with Progressing Ischemic Stroke. Journal of the Neurological Sciences, 163, 86-89. http://dx.doi.org/10.1016/S0022-510X(98)00267-6

[7] Dupont, S., Catala, M., Hasboun, D., Semah, F. and Baulac, M. (1997) Progressive Facial Hemiatrophy and Epilepsy: A Common Underlying Dysgenetic Mechanism. Neurology, 48, 1013-1018. http://dx.doi.org/10.1212/WNL.48.4.1013

[8] Chung, J.M. (2014) Seizures in the Acute Stroke Setting. Neurological Research, 36, 403-406. http://dx.doi.org/10.1179/1743132814Y.0000000352

[9] Chung, M.H., Sum, J., Morrell, M.J. and Horoupian, D.S. (1995) Intracerebral Involvement in Scleroderma en Coup de Sabre: Report of a Case with Neuropathologic Findings. Annals of Neurology, 37, 679-681.

http://dx.doi.org/10.1002/ana.410370519 